\title{
An Elementary Solution for a Difficult Motion Problem
}

Daniel A. Martínez and Santiago L. Queiro

Citation: The Physics Teacher 41, 518 (2003); doi: 10.1119/1.1631620

View online: http://dx.doi.org/10.1119/1.1631620

View Table of Contents: http://aapt.scitation.org/toc/pte/41/9

Published by the American Association of Physics Teachers

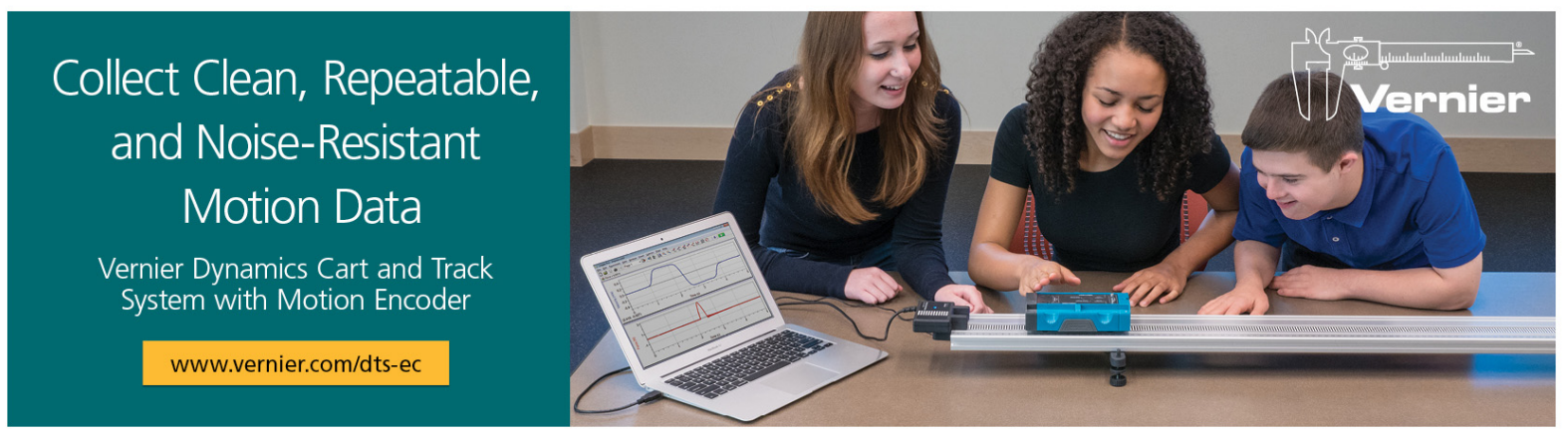




\section{An Elementary Solution for a Difficult Motion Problem}

\section{Daniel A. Martínez and Santiago L. Queiro, Universidad de Buenos Aires, Argentina}

\section{Essentially geometrical arguments are applied to solve a seemingly unsolvable problem.}

\section{Formulation of the problem}

A wet umbrella rotates with an angular velocity $\omega$ with its axis parallel to the ground.

a) Draw the trajectories followed by three arbitrary drops that escape from the rim of the umbrella.

b) From which point of the umbrella's rim must a drop be ejected to have a maximum horizontal range?

c) From which point of the umbrella's rim must a drop be ejected to reach the maximum height?

This problem is one of the exercises from the final chapter of the introductory noncalculus-based book ${ }^{1}$ on mechanics used since 1991 in the CBC of Buenos Aires University. Part a) of the exercise was proposed to detect false concepts in trajectories. Parts b) and c) are indicated as difficult ones and only a qualitative answer is required from students.

At first glance, many students (and teachers) suggest that a drop detached from the rim forming a $45^{\circ}$ angle to the vertical axis will reach the furthest point on the horizontal, but a careful analysis shows that a drop ejected from a higher point will reach further because it starts closer to the axis.

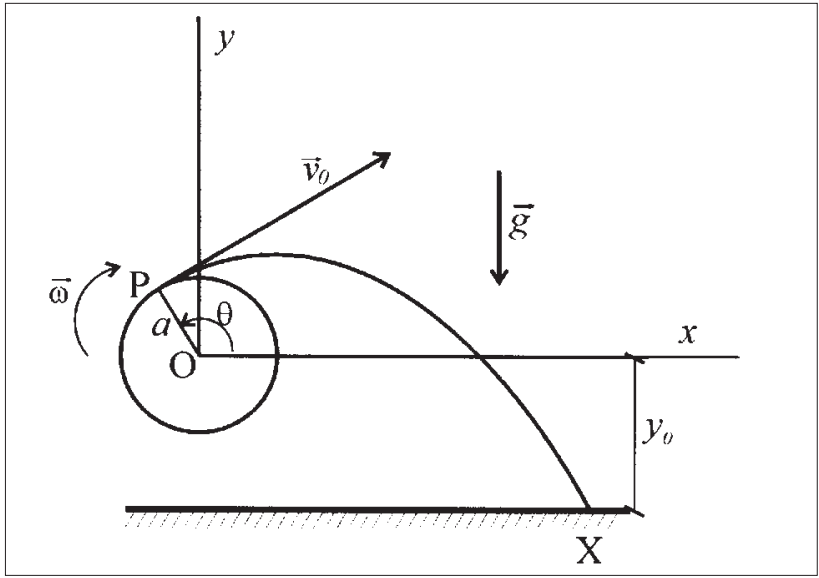

Fig. 1. The circle represents a rotating umbrella with its axis horizontally fixed at a height $y_{0}$ from the ground. A drop is ejected with velocity $\vec{v}_{0}$ from a point $P$ on the umbrella's rim and reaches the floor at $X$. Where must $P$ be to get the maximum range?

\section{Equations of Motion in a Cartesian Coordinate System}

Suppose that the umbrella is represented by a circle of radius $a$, and its axis is horizontally fixed at a height $y_{0}$ from the ground. Let us consider a drop ejected with velocity $\vec{v}_{0}$ from a point $\mathrm{P}$ on the umbrella's rim as shown in Fig. 1. The components of the drop's position vector in the Cartesian coordinate system of Fig. 1 are:

$$
\begin{aligned}
& x(t, \theta)=a \cos \theta+v_{0} \sin \theta t \\
& y(t, \theta)=a \sin \theta-v_{0} \cos \theta t-\frac{1}{2} g t^{2} .
\end{aligned}
$$


Equations (1) and (2) can be written in a condensed form using the expression for the position vector:

$$
\vec{r}(t)=\vec{r}_{0}+\vec{v}_{0} t+\frac{1}{2} \vec{g} t^{2}
$$

with $\vec{r}_{0}=a \cos \theta \hat{i}+a \sin \theta \hat{j}$.

\section{Maximum Horizontal Range}

Maximizing the horizontal range $x$ implies the use of advanced mathematical tools such as Lagrange's multipliers, since variables $\theta$ and $t$ are entangled in both Eqs. (1) and (2). This method far exceeds the basic math background needed for the course. However, for fixed values of parameters $y_{0}, a, v_{0}$, and $g$ it is possible to calculate the angle $\theta$ that maximizes the horizontal range using geometrical arguments.

For simplicity we shall consider the following two cases separately:

Case A) The drop is thrown to the right with an angle $\theta$.

Case B) The drop is thrown to the left with an angle $\theta^{*}$, Fig. 2.

\section{Case A)}

A useful vector-addition diagram representing Eq. (3) is shown in Fig. 3. This construction is similar to that employed in many introductory level physics books. ${ }^{2}$ Particle motion can be analyzed by considering a displacement PQ with constant velocity $\vec{v}_{0}$, followed by a vertical fall $Q R$ with acceleration $\vec{g}$. Figure 4 shows that OQ is the common hypotenuse of the two right-angle triangles ORQ and OPQ. Then the following relation stands for the lengths of the other sides:

$$
a^{2}+v_{0}^{2} t^{2}=x^{2}+\frac{1}{4} g^{2} t^{4}-g y_{0} t^{2}+y_{0}^{2} .
$$

From Eq. (4) an expression for $x^{2}$ in function of time can be obtained:

$$
x^{2}(t)=-\frac{1}{4} g^{2} t^{4}+\left(v_{0}^{2}+g y_{0}\right) t^{2}+\left(a^{2}-y_{0}^{2}\right) .
$$

It can be seen that $x^{2}$ is a quadratic function in $t^{2}$ with a maximum value for: ${ }^{3}$

$t_{\mathrm{M}}^{2}=\frac{2\left(v_{0}^{2}+g y_{0}\right)}{g^{2}}(6)$ or $t_{\mathrm{M}}=\frac{\sqrt{2\left(v_{0}^{2}+g y_{0}\right)}}{g}$.

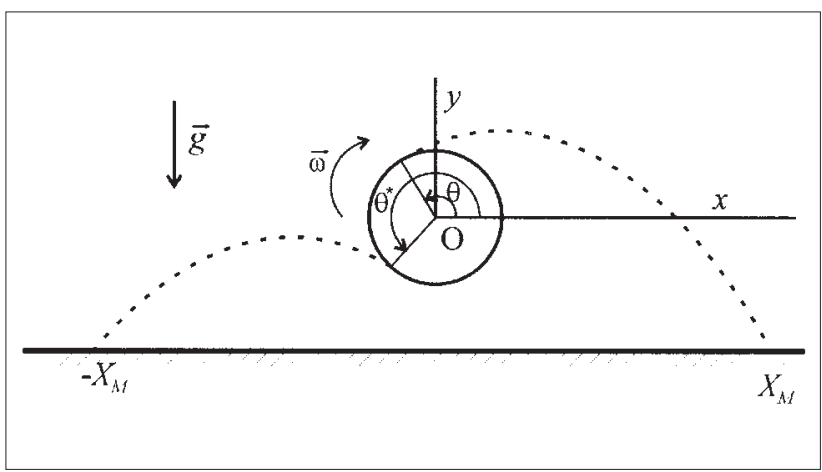

Fig. 2. Trajectories followed by two drops thrown with polar angles $\theta$ and $\theta^{*}$, reaching the farthest points to the right and to the left.

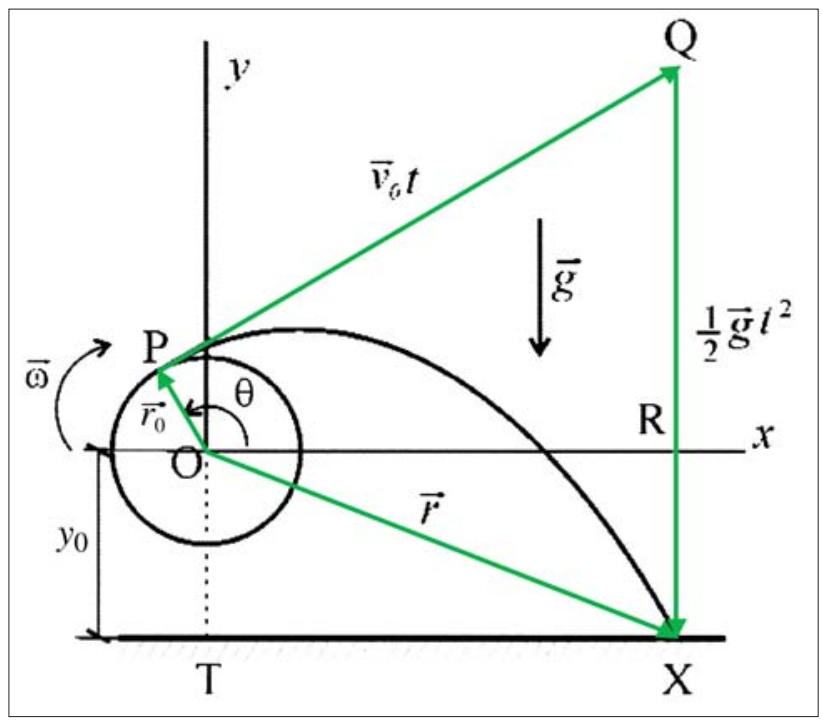

Fig. 3. Vector-addition diagram representing Eq. (3).

Maximum range is obtained by substituting Eq. (6) in Eq. (5):

$$
X_{\mathrm{M}}= \pm \frac{\sqrt{v_{0}^{4}+2 v_{0}^{2}} \overline{g y_{0}}+\bar{g}^{2} a^{2}}{g},
$$

where the plus sign in Eq. (8) gives the maximum horizontal range to the right of the umbrella and the minus sign the corresponding value to the left.

From triangles ORQ and OPQ of Fig. 4, and the expressions of $t_{\mathrm{M}}$ and $X_{\mathrm{M}}$ given by Eqs. (7) and (8), the following relations for the angles $\alpha$ and $\beta$ can be obtained:

$$
\alpha=\operatorname{atan}\left[\frac{v_{0}^{2}}{\sqrt{\mathrm{v}_{0}^{4}+2 v_{0}^{2}} \overline{g y_{0}+g^{2} a^{2}}}\right]
$$




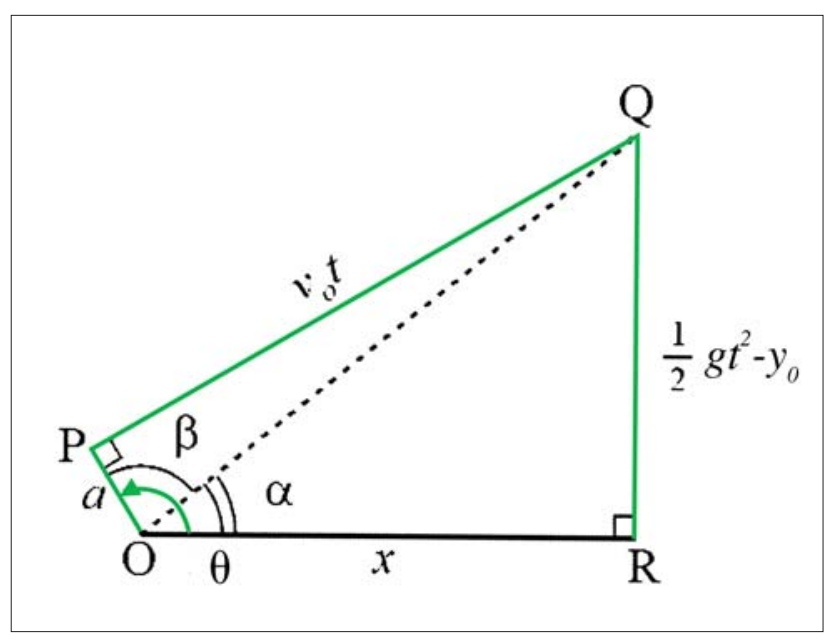

Fig. 4. Right angle triangles $O R Q$ and $O P Q$ with a common hypotenuse $O Q$. Maximum range to the right is obtained maximizing side $x$.

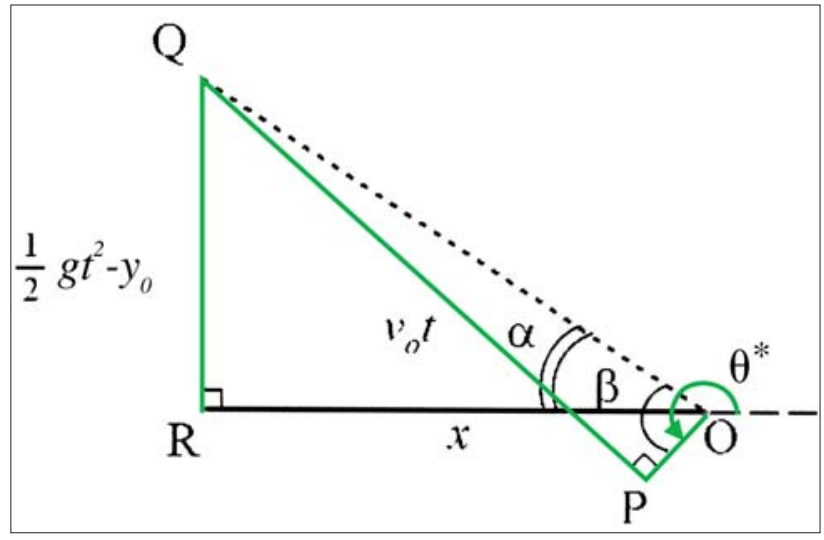

Fig. 5. Right angle triangles $O R Q$ and $O P Q$ with a common hypotenuse $O Q$. Maximum range to the left is obtained maximizing side $x$.

$$
\beta=\operatorname{atan}\left[\frac{\sqrt{2 v_{0}^{2}\left(v_{0}^{2}+g y_{0}\right)}}{g a}\right] .
$$

From Fig. 4 the angle for maximum range to the right is then:

$$
\theta=\alpha+\beta \text {. }
$$

\section{Case B)}

Figure 5 shows the corresponding triangles ORQ and OPQ for a drop ejected to the left. The value of the angles $\alpha$ and $\beta$ are given by expressions (9) and (10) again, since the magnitude of the maximum range to both sides is the same as can be seen from Eq. (8). Angles $\theta^{*}, \alpha$, and $\beta$ are related in this case by:

$$
\theta^{*}=\beta-\alpha+\pi
$$

For typical values of $g=9.8 \mathrm{~m} / \mathrm{s}^{2}, y_{0}=1 \mathrm{~m}$, $a=0.5 \mathrm{~m}$, and $v_{0}=5 \mathrm{~m} / \mathrm{s}$, one obtains:

$X_{\mathrm{M}} \cong \pm 3.44 \mathrm{~m}, \theta \cong 120^{\circ}$, and $\theta^{*} \cong 227^{\circ}$.

We leave the reader to prove that the angle from which a drop leaves the rim reaching the maximum height is given by:

$$
\begin{aligned}
& \theta_{\mathrm{H} \max }=\arcsin \left(\frac{a g}{v_{0}^{2}}\right) \text { for } \theta_{\mathrm{H} \max } \geq \frac{\pi}{2} \text { and } v_{0}^{2} \geq a g . \\
& \theta_{\mathrm{H} \max }=\frac{\pi}{2} \quad \text { for } \quad v_{0}^{2}<a g .
\end{aligned}
$$

\section{Acknowledgment}

The authors wish to thank the referee for his/her critical comments, which have helped to bring this work to its final form.

\section{References}

1. A.M. Rela et al., Física UBA CBC (Gráfica Yanel, 2000), p. 80.

2. R. Resnick, D. Halliday, and K. Krane, Physics, 4th ed. (Wiley, New York, 1992), p. 56.

3. Of course, this maximum value can be obtained from Eq. (9) differentiating $x^{2}(t)$ with respect to $t^{2}$ and proceeding in the usual way. But it is interesting to note that this problem can be solved without use of calculus.

PACS codes: $02.40,46.01 \mathrm{~A}$

Daniel A. Martínez is Licenciado en Física from Universidad de Buenos Aires. He has taught high school physics for more than 20 years. Currently, he is an assistant professor of physics at the Ciclo Básico Común of Universidad de Buenos Aires and at Universidad Tecnológica Nacional. His research interests include teaching methods in physics and soil physics.

Santiago L. Queiro is an electronics engineer from Universidad de Buenos Aires. He has taught physics for more than 30 years. Currently, he is professor of physics at the Ciclo Básico Común of Universidad de Buenos Aires and at Universidad Tecnológica Nacional. His research interests include teaching methods in physics with developing experiments for engineering and life sciences.

Ciclo Básico Común, Universidad de Buenos Aires, Pabellón III, Ciudad Universitaria, 1428 Buenos Aires, Argentina; danmarbaum @fibertel.com.ar, squeiro@ hotmail.com 